\title{
La afectividad, el autoconcepto y las relaciones interpersonales en agresores sexuales intrafamiliares a través del psicodiagnóstico de Rorschach
} \author{
the Rorschach psichodiagnostic \\ Juan Artica M. '; GLoria DíazA. \\ Universidad Nacional Mayor de San Marcos, Lima, Perú \\ (RECIBIDO 18-08 2014, ACEPTADO 20-10-2014)
}

Emotion, self-concept and relationships in intrafamily sex offenders through

\begin{abstract}
RESUMEN
La presente investigación es de tipo descriptiva, se realizó con el propósito de conocer los rasgos afectivos, el autoconcepto y las relaciones interpersonales en agresores sexuales intrafamiliares, para tal fin se aplicó el Psicodiagnóstico de Rorschach y una encuesta que recogió datos sociodemográficos e información relacionada a la agresión sexual cometida por los participantes. La muestra está conformada por 30 internos de un establecimiento penitenciario, identificados por haber cometido delito contra la libertad sexual-abuso sexual intrafamiliar. Los resultados obtenidos develan que el grupo de agresores sexuales intrafamiliares se caracteriza por presentar escasos recursos para controlar descargas afectivas intensas, respondiendo de manera impulsiva ante este tipo de situaciones. Asimismo, se observa una autovaloración impregnada de matices de autocrítica negativa, lo que genera una estrategia defensiva de tipo narcisista para negar el dolor y la limitación. Igualmente, se aprecia estados de constricción emocional, es decir, un freno a la expresión emocional, así como una tendencia a centrase en sí mismos más de lo habitual, lo que les dificulta ver las cosas desde otras perspectivas. Este grupo presenta dificultades para establecer relaciones interpersonales profundas y significativas.
\end{abstract}

Palabras clave: Personalidad, autoconcepto, relaciones interpersonales, afectos, agresor sexual intrafamiliar.

1 PsicólogoporlaUniversidadNacional MayordeSanMarcos. EstudiantedelaMaestríaenPsicologíaClínicay delaSalud. E-mail:juanartica@gmail.com 


\begin{abstract}
This descripted research was performed with the objective of knowing the affective traits, self-concept and interpersonal relationships in intrafamiliar sex offenders. For that purpose, it was applied the Rorschach Psycodiagnostic and a survey that collected demographic data and information on sexual assault committed by the participants. The sample consists of 30 inmates of a correctional facility, identified as having committed sexual crimes against sexual freedom and domestic abuse. According to the results, it is disclosed that the group of intrafamiliar sex-offenders are characterized by scarce resources to control intense emotional shock; responding impulsively in these situations. Also, a self-assessment is impregnated of nuances of negative self-observed, generating such a defensive devaluation of narcissistic strategy to deny the pain and limitation. Similarly, emotional constriction states are shown, that is, there is a poor emotional expression. On the other hand, it is shown a tendency to focus on themselves more than usual, making it difficult to see things from other perspectives, also presents difficulties to establish deep and meaningful relationships.
\end{abstract}

Keywords: Personality, self-concept, interpersonal relationships, affection, family sexual aggressor.

\title{
INTRODUCCIÓN
}

La Declaración Universal de los Derechos Humanos señala: “...la libertad, la justicia y la paz en el mundo tienen por base el reconocimiento de la dignidad intrínseca y de los derechos iguales e inalienables de todos los miembros de la familia". Esta afirmación le atribuye al núcleo de la sociedad la responsabilidad de ser el escenario que genere las condiciones necesarias para el reconocimiento del otro. Sin embargo, en un plano real, diversos reportes científicos y periodísticos denotan que la familia se ha convertido en el escenario más sensible de la génesis de la agresión contra el otro. Esto permite ver el rostro más crudo de la realidad, en el que la violencia -en cualquiera de sus manifestaciones-irrumpe y desteje los vínculos de amor y confianza que resquebrajan las bases de la convivencia armónica.

La problemática de la violencia intrafamiliar no es un tema moderno, pues durante años el daño se ha mantenido oculto y encubierto, generando progresivamente una espiral interminable. Generaciones de padres y madres han usado el maltrato en contra de sus hijos (niños, niñas y adolescentes) como una forma de crianza y/o corrección; pensando quizás que sería la mejor manera de educarlos. Estas concepciones ético-morales, propias del modelo tradicional, mantenidas aún hasta la actualidad, sustentan una educación vertical y represora, de obediencia, sumisión y "respeto", que solamente desarrolla mayor agresividad en los propios hijos, surgiendo así, sentimientos de hostilidad, resentimiento y rencor hacia lo humano. Lo último se agudiza aún más cuando los menores son víctimas de abuso sexual dentro de sus mismos "hogares".

El maltrato infantil, en su variedad de expresiones, presenta su punto más álgido en el abuso sexual infantil, siendo más común de lo que se piensa. Se reconoce que en la historia de la humanidad han existido violaciones dentro del ámbito familiar; sin embargo, la frecuencia con las que se identifican y registran varía 
notablemente (Viviano, 2012). Por eso, tener una cifra confiable y exacta sobre esta problemática es muy difícil, sobre todo para los casos de abuso sexual intrafamiliar como los que se han estudiado para la presente investigación, porque resulta complicado para la población identificar la situación y luego denunciar el hecho. Las afectadas, en su mayoría mujeres, no denuncian haber sido agredidas sexualmente para evitar una segunda victimización o porque se sienten avergonzadas parcialmente responsables de lo sucedido.

Aun cuando no existe un registro completo, las cifras disponibles son preocupantes no solo en Lima, sino también en el interior del país donde esta problemática social y de salud, según la Organización Mundial de la Salud (OMS), se incrementa día a día. En 2013, 5550 casos de abuso sexual han sido atendidos por los Centros Emergencia Mujer (CEM), 4265 de ellos corresponden a niños, niñas y adolescentes entre 0 y 17 años de edad; es decir, el $76.84 \%$ de la población atendida agrupa a la infancia y adolescencia (MIMP, 2013). Asimismo, según el reporte de 2012 emitido por los CEM, el 51\% de las personas afectadas por violencia familiar y sexual señalaron que el agresor residía en su misma vivienda. Para el caso de abuso sexual intrafamiliar, las figuras del padre, padrastro y abuelos destacan como los principales agresores (MIMP, 2012).

Frente a esta situación, las instituciones gubernamentales y no gubernamentales se encuentran formulando y ejecutando acciones en pro de la reducción de estos casos. Del mismo modo, se han realizado numerosas investigaciones en torno al tema; no obstante, dichos estudios de carácter descriptivo y explicativo se han centrado en la figura de la víctima, restando interés a otro personaje no menos importante dentro del escenario de abuso: el agresor. Por ello, ante esta necesidad de conocimiento, la presente investigación pretende contribuir a develar información relevante entorno a esta problemática.

Si consideramos los estudios de carácter psicológico, criminológico, antropológico y sociológico de los agresores, podemos advertir que el agresor sexual de menores no es un psicópata propiamente dicho: no sufre esa alteración tan profunda, pero sí tiene una personalidad con rasgos peculiares, los mismos que se describen en este estudio.

Marshall y Barbaree (1990, como se citó en Garrido, 2005) elaboraron un modelo comprensivo de la etiología de la agresión sexual, donde tenían cabida las influencias biológicas, el contexto sociocultural y el desarrollo psicológico del individuo. En referencia a este último elemento, señalaban la presencia de sentimientos de inferioridad y falta de competencia social; además, la tendencia a recurrir al sexo como estrategia para enfrentar situaciones de estrés, lo que propiciaba el deseo de agredir sexualmente en tales circunstancias. Según esta teoría, el sexo se convertiría en el modo predilecto de reaccionar cuando el individuo se siente angustiado amenazado. ¿Qué más podría propiciar este tipo de agresión? Estos aportes motivaron y alimentaron el interés en conocer con mayor precisión aquellas características de personalidad relevantes en el agresor sexual, por eso el propósito del estudio fue describir los rasgos afectivos, el autoconcepto y las relaciones interpersonales en un grupo de agresores sexuales intrafamiliares. 


\section{MÉTODO}

Según la clasificación de Hernández, Fernández y Baptista (2010) la presente investigación tiene un alcance descriptivo y su diseño es no experimental de tipo transeccional. Por otro lado, respecto a la muestra, ésta responde a un muestreo de tipo no probabilístico propositivo (Kerlinger, 2002). Así, para responder a los objetivos de esta investigación, se empleó una muestra de 30 varones adultos, padres y padrastros, que cometieron abuso sexual contra sus hijas y entenadas respectivamente. Estos participantes se encuentran, actualmente, recluidos en el pabellón 16 del penal de San Juan de Lurigancho.

Los criterios de selección de la muestra fueron los siguientes:

1. Ser sentenciado por el delito de violación de la libertad sexual de acuerdo con el Art. 173 del Código Penal (violación sexual de menor de edad).

2. Haber agredido sexualmente a su hija o entenada.

3. Reconocer su responsabilidad en la agresión sexual hacia su hija o entenada.

4. No tener antecedentes psiquiátricos.

Dentro de la muestra, las edades de los agresores al momento de la investigación oscilaban entre los 39 y 62 años de edad (Me=49.40, DE=6.81); sin embargo, las edades en las que ocurrió la agresión sexual intrafamiliar fue entre los 29 y 56 años ( $M e=40.37, \mathrm{DE}=6.69$ ). Asimismo, la mayor distancia en tiempo entre el momento de la agresión sexual intrafamiliar y el momento de la investigación fue de 10 años y la menor fue de 6 años.

\section{Instrumentos}

A fin de cumplir con los objetivos de investigación se utilizaron dos instrumentos:

Encuesta de datos sociodemográficos y del episodio de agresión sexual intrafamiliar

Se construyó una encuesta que, para los fines de la investigación, se dividió en dos áreas: el Área I que consigna los datos sociodemográficos del participante como la edad al momento de la encuesta, lugar de nacimiento, grado de instrucción, estado civil, ocupación y un código que reemplaza al nombre del participante; y el Área II que consigna datos biográficos de la agresión sexual.

\section{Psicodiagnóstico de Rorschach}

Se utilizó además el Psicodiagnóstico de Rorschach según el Sistema Comprehensivo de Exner, prueba proyectiva que mide la personalidad. Dentro de este sistema creado por Exner (2005) los indicadores relevantes para la presente investigación son: las agrupaciones o secciones de rasgos afectivos, autopercepción y relaciones interpersonales. A la vez cada uno de éstos tiene sus propios indicadores, los mismos que se describen a continuación: 


\section{Rasgos afectivos}

- Índice de depresión (DEPI) e Índice de inhabilidad social (CDI): aunque el DEPI, dada su relevancia diagnóstica funciona en sí mismo como una variable clave desde la cual se puede comenzar la interpretación de un protocolo, es conveniente revisar sus referencias como dato emocional, ya que incluye una serie de variables afectivas, además de otras cognitivas y relacionales, e indica un trastorno afectivo importante cuando aparece como positivo. Por otro lado, el CDI se verá más extensamente en la agrupación de relaciones interpersonales, por tratarse de un elemento nuclear del mismo.

- Lambda, EB extratensivo y EBPer: el hecho de que un L señale un estilo evitativo matiza el significado del EB extratensivo. La presencia de ambos datos en un protocolo apunta a que el sujeto es un extratensivo- evitativo, es decir, tiende a no diferenciar claramente las características e implicaciones de los contextos emocionales complejos, a no regular la influencia de los sentimientos en sus procesos de toma de decisión y a no preocuparse demasiado por la modulación de sus descargas afectivas, debido a su inclinación a simplificar el análisis de las situaciones. Por otro lado, el EBPer, cuando es positivo, indica una rigidificación del estilo básico de respuesta que restará eficacia adaptativa de las conductas.

- Análisis del lado derecho de la eb: consiste en una revisión del lado derecho de la experiencia base (eb) y de las variables que con él se relacionan, para averiguar si el sujeto está sufriendo una experiencia inusual de malestar.

- Relación SumC': SumPondC. Esta proporción está relacionada con la supresión o la contención de las emociones.

- Proporción afectiva (Afr): esta variable expresa el interés del sujeto por experimentar sensaciones o rodearse de emociones.

- Índice de intelectualización $(2 A b+A r t+A y)$ : proporciona información relacionada con el uso de la intelectualización. Es un proceso mediante el cual el impacto de las situaciones cargadas de emoción o de las vivencias emocionales se ve reducido, o incluso neutralizado, manejándolas de manera ideacional más que emocional.

- Proporción del color (CP): las proyecciones del color son muy raras y se espera que el valor CP sea siempre cero. La presencia de una sola CP ya tiene una especial importancia interpretativa, dado que implica una forma insólita de negación cuando se procesan experiencias emocionales desagradables.

- Proporción forma- color $(F C$ : $C F+C)$ y presencia del color puro (C Pura): la proporción $\mathrm{FC}$ : $\mathrm{CF}+\mathrm{C}$ y el valor de $\mathrm{C}$ pura proporcionan información relacionada con la modulación de la descarga o de la expresión emocional. Por otro lado, las respuestas de $C$ pura estima de forma subjetiva hasta qué punto representan un tipo de respuestas menos maduras o más primitivas en comparación con aquellas que reflejan una mayor contención. El grado de refinamiento de las respuestas de C pura varía de manera considerable.

- Respuestas de espacio blanco (S): si la cantidad de respuestas S es excesiva habrá que considerar las opciones del negativismo, el oposicionismo o incluso el enfado. 
- Composición y cualidad de las Respuestas complejas (Complj), Relación con EB y Lambda. Respuestas complejas por M o Y. Complejas de Color- Sombreado (Complj. Col-SH) y de Sombreado- Sombreado (Complj.SH): en las respuestas complejas el sujeto trabaja con más de un determinante simultáneamente, desarrollando una actividad cognitiva más elaborada y difícil que la requerida para cumplir la consigna de la prueba. En este sentido, estas respuestas son lo opuesto a las respuestas de forma pura. Mientras éstas denotan una clasificación simple y directa de los datos, aquellas son producto de una considerable actividad de análisis y síntesis de los elementos del campo a estimular.

\section{Autopercepción}

- Índice obsesivo (OBS) e índice de hipervigilancia (HVI): el OBS tiene que ver con la meticulosidad, el perfeccionismo y la eficacia y el HVI con la preocupación por proteger la integridad personal, sensación de debilidad y un marcado sentimiento de vulnerabilidad.

- Índice de egocentrismo $(3 r+(2) / R)$ y reflejos $(F r+r F)$ : el índice de egocentrismo se relaciona con la autoestima y los reflejos con componentes narcisistas, con tendencia a la sobreestima personal.

- Respuestas de vista y forma dimensión (V y FD) en relación con la historia personal: las primeras tienen que ver con los aspectos negativos o displacenteros de la autoimagen y las segundas con la capacidad de introspección.

- Contenidos anatómicos y radiografía $(A n+X y)$ : los dos contenidos se relacionan con la preocupación por el cuerpo y uno mismo, siendo el contenido radiografía (Xy) más racional.

- MOR y contenidos asociados: los contenidos mórbidos (MOR) reflejan una posición depresiva y un proceso ideacional particularmente negativista.

- Proporción $\mathrm{H}:(\mathrm{H})+\mathrm{Hd}+(\mathrm{Hd})$; revisión de la calidad formal (FQ) y los contenidos de la categoría H; GHR:PHR: en la proporción debe predominar la H sobre la $(H), H d$ y $(\mathrm{Hd})$ para poder inferir que la percepción de uno mismo está basada en la realidady no en la fantasía, la revisión de la FQ y los contenidos H brindan información acerca de la calidad de los contenidos humanos y la representación humana buena (GHR) y la pobre (PHR) dan información acerca de la presencia de aspectos adaptativos o distorsionadores, respectivamente, que se introducen a las respuestas de contenido humano.

- Búsqueda de proyecciones en respuestas con FQ-, respuestas MOR, respuestas de movimientos y sobre elaboraciones verbales: estas respuestas proporcionan con mayor probabilidad atribuciones personales que no vienen dadas por el campo a estimular. Las respuestas con FQ- son aquellas que se apartan de lo convencional; el MOR tiene que ver con impresiones negativas que la persona tiene sobre si misma; las respuestas de movimiento hablan de elementos personales que se atribuyen a los objetos y dan una información importante en relación al autoconcepto. Importante analizar en primer lugar 
las respuestas de movimiento humano (M), luego las de movimiento animal (FM) y por último las de movimiento inanimado $(\mathrm{m})$. Finalmente, las respuestas de elaboraciones verbales tienen que ver con el material proyectivo que ofrece información sobre la autoimagen de la persona evaluada.

\section{Relaciones interpersonales}

- Índice de inhabilidad social (CDI) e índice de hipervigilancia (HVI): el CDI representa las dificultades para manejarse e interactuar en las relaciones sociales y el HVI se refiere a una actitud negativista y desconfiada hacia el entorno.

- Relación activo: pasivo (a: p): tiene que ver con asumir un rol más pasivo o activo en el intercambio interpersonal.

- Respuestas de comida ( $\mathrm{Fd}$ ) y textura ( $\mathrm{T}$ ): las respuestas $\mathrm{Fd}$ reflejan las necesidades orales y dependientes del sujeto y las $\mathrm{T}$ la búsqueda o necesidad de la proximidad emocional en las relaciones interpersonales.

- Análisis de los contenidos humanos: aporta información acerca de la capacidad de la persona a experimentar vínculos reales basados en la experiencia versus vínculos irreales, además sobre la capacidad de adaptación de la persona dentro de sus relaciones interpersonales y dentro de su medio.

- GHR:PHR: las respuestas GHR hablan de mayor capacidad para adaptarse a las relaciones interpersonales y por ende tener un manejo más eficaz de las mismas y las PHR hablan mas bien de relaciones interpersonales poco favorables, que se quedan en el plano de la fantasía y que van a redundar en alteraciones en la autoimagen.

- Revisión de los códigos personal (PER), cooperativo (COP) y agresivo (AG): las respuestas COP se relacionan con el cómo se vivencian los vínculos solidarios que uno es capaz o no de construir; las respuestas PER tienen que ver con una necesidad de justificar y reforzar defensivamente la autoimagen para protegerse de un supuesto cuestionamiento del otro y las respuestas AG se relacionan con la posibilidad de entender el vínculo con los demás desde lo agresivo y la disputa.

- Índice de aislamiento (Aislamiento/R): se relaciona con el aislamiento social. Se compone de los contenidos de cinco categorías (Botánica, Nubes, Geografía, Paisaje y Naturaleza).

- Contenidos de M y FM con pares: proporciona información acerca de las características que el sujeto destaca cuando establece vínculos interpersonales.

\section{DISCUSIÓN Y RESULTADOS}

\section{Indicadores de la sección principal}

La Tabla 1, en primer lugar, presenta el número de respuestas $(R)$ y el valor de Lambda (L), dos variables necesarias para la comprobación de la validez de los protocolos Rorschach (Sendín, 2007). 
Tabla 1. Indicadores relevantes de la sección principal.

\begin{tabular}{lcc}
\hline & $\mathrm{M}$ & $\mathrm{DE}$ \\
\hline $\mathrm{R}$ & 20.73 & 3.64 \\
Lambda & 0.51 & 0.21 \\
EA & 4.38 & 2.28 \\
Es & 11.8 & 3.55 \\
Adj es & 11.43 & 3.82 \\
D & -2.4 & 1.58 \\
Adj D & -2.2 & 1.72 \\
EB & $1.87: 2.52$ & $1.26: 2.20$ \\
Eb & $5.73: 6.07$ & $2.19: 5.23$ \\
\hline
\end{tabular}

Fuente: De la muestra de colaboradores del estudio.

Luego se muestran los indicadores con valores significativos como la Experiencia Accesible (EA), Estimación Sufrida (es), Tolerancia al Estrés Crónico (Adj es), Puntuación D (D), Puntuación D Ajustada (Adj D), Erlebnistypus (EB) y la Experiencia base (eb).

Respecto al número de respuestas $(R)$, se encuentra una media 20.73 ( $\mathrm{Me}=20.73$ ) en el grupo de estudio, este valor es mayor al obtenido en otro estudio realizado en Chile con una población similar, compuesta por 20 individuos condenados por delito sexual (Jiménez, 2009).

En relación a la Lambda $(\mathrm{L})$, se obtuvo une media de 0.51 ( $\mathrm{Me}=0.51$ ), este valor es menor al obtenido en el estudio chileno mencionado líneas arriba. Esta puntuación muestra un nivel adecuado de apertura a referir aspectos emocionales e ideacionales; es decir, existe un esfuerzo de los evaluados por hablar de sí mismos. Además, no se muestran defensivos y no tienden a sobre simplificar la información de la realidad.

Ambas puntuaciones confirman la validez de los protocolos utilizados en esta investigación.

La experiencia accesible (EA) en este estudio tiene una media de 4.38 ( $\mathrm{Me}=$ 4.38). Esta variable resulta de la Suma de Movimiento Humano (M) más la Suma Ponderada de Color Cromático (SumPondC=0.5xFC+1.0xCF+1.5xC), y constituye un indicador de los recursos disponibles con los que cuenta el sujeto para iniciar conductas deliberadas; es decir, del bagaje potencial para tomar decisiones y ponerlas en práctica (Sendín, 2007). Este dato estaría reflejando la poca cantidad de recursos disponibles en los evaluados para afrontar situaciones de tensión.

Se observa un incremento de las variables afectivas $\left(C^{\prime}+V+T+Y\right)$ en el indicador de Estimulación Sufrida (es), lo cual hace referencia a la presencia de síntomas situacionales, pero que también están instalados en el funcionamiento ordinario y aporta un potencial considerable de impulsividad al pensamiento, los afectos y 
la conducta (Sendín, 2007). Los individuos con este dato están más expuestos a desorganizarse ante situaciones complejas, riesgo que se aumenta aún más por su baja puntuación en la EA.

Por otro lado, el EB es ambiguo, lo cual indica una mayor indefinición a la hora de enfrentarse a la solución de problemas; asimismo, convierte su conducta en más errática e imprevisible (Sendín, 2007). Este valor es similar al obtenido en el estudio chileno (Jiménez, 2009).

La experiencia base (eb) indica una puntuación ligeramente mayor del lado derecho de la eb, la misma que está relacionada con afectos irritativos o perturbadores; asimismo, se observa que la puntuación de respuestas vista (Sum V) es mayor frente a las demás puntuaciones de este lado de la eb. Esto señala la existencia de fuertes componentes de desvalorización asociados a procesos introspectivos inadecuados. Indica que cuando el sujeto realiza tareas de autoexamen, las impregna de matices de autocrítica negativa, produciéndose, consecuentemente, sentimientos de desagrado y rebeldía, que aumentan el sufrimiento psíquico (Sendín, 2007).

\section{Indicadores del clúster de rasgos afectivos}

La siguiente tabla muestra los indicadores que conforman el clúster de rasgos afectivos, elementos más complejos, móviles e inasequibles del psiquismo humano (Sendín, 2007).

La presencia de EB ambigual (observado en tabla 2), señala que los evaluados se muestran más ineficientes, cometen más errores y consiguen menos aciertos. Además, no tienen un estilo definido de respuesta básica y por ello, procesan afectos superficiales, siendo mucho más impredictibles en su conducta. No obstante, en ocasiones sus procesos de pensamiento se verán impregnados de emoción, jugando estas un papel primordial.

Tabla 2. Indicadores del clúster de rasgos afectivos.

\begin{tabular}{lcc} 
& M & DE \\
\cline { 2 - 3 } Sum C' & 1.77 & 1.75 \\
Sum T & 0.43 & 0.88 \\
Sum V & 3.67 & 2.2 \\
Sum Y & 0.2 & 0.4 \\
FC:CF+C & $1.47: 1.30$ & $1.50: 1.66$ \\
CPura & 0.97 & 1.17 \\
Sum C' & 1.77 & 1.75 \\
WSum C & 2.52 & 2.2 \\
Afr & 0.6 & 0.26 \\
S & 1.07 & 1.03 \\
Complejas: & 0.12 & 0.1 \\
\hline
\end{tabular}

Fuente: De la muestra de colaboradores del estudio. 
Al observar el lado derecho de la eb $\left(C^{\prime}+T+V+Y\right)$, los sombreados y el color acromático resaltan sobre los determinantes de movimiento no humano $(F M+m)$, señalando la influencia de los afectos en la conducta. Esto evidencia que los evaluados tienen escaso control sobre sus impulsos y que al incrementarse estos valores sobrepasan el monto de recursos organizados con los que cuentan y provocan una situación de sobrecarga que predispone a las conductas impulsivas sostenidas alejadas del control ideacional.

El color acromático $\left(C^{\prime}\right)$ con una puntuación mayor a 1 (Sum $\left.C^{\prime}=1.77\right)$, indica la presencia inconsistente de constricción afectiva; es decir, un freno precario de la expresión emocional. El grupo evaluado en lugar de registrar un alivio por la externalización o descarga de algún afecto, lo interioriza, no permite su salida y con ello aumenta su incomodidad interior. Cabe señalar que $C^{\prime}$ es involuntario y automático (Sendín, 2007).

En las respuestas de textura $(T)$, se encuentra una medida de 0.43 ( $\mathrm{Me}=0.43$ ), la cual indica un adecuado manejo de cercanía; es decir, los evaluados no se muestran distantes en sus contactos con los demás y muestran una actitud de cálculo en el pensamiento.

Las puntuaciones Sum $V(\mathrm{Me}=3.67)$ y la suma de respuestas reflejas $(\mathrm{Fr}+\mathrm{rF}$ $>0$ ) indican actividades ambivalentes donde por un lado el grupo evaluado se critica muy negativamente y por otro, tiende a sobrevalorarse. Lo cual se explica que en estos casos puede ocurrir que la alta desvalorización genere una estrategia defensiva de tipo narcisista para negar el dolor que su conducta genera en otros (Sendín, 2007).

El valor de Sum $Y$ es bajo lo cual indica una menor tendencia a experimentar o reconocer estados de ansiedad. Similar valoración se encontró en un estudio de delincuentes sexuales (Jiménez, 2009).

La relación SumC': WSumC indica que el lado izquierdo de esta relación es inferior al derecho; por lo tanto, el nivel de procesamiento de emociones deliberadas que el sujeto habitualmente utiliza es mayor al grado de constricción afectiva. Lo cual significa que estos evaluados tienen incapacidad para asumir introspectivamente la valoración de sus actos.

Respecto a la Proporción afectiva (Afr), se encuentra una media de 0.60 (Me = 0.60 ) en el grupo de estudio, lo cual indica un menor interés por procesar estímulos afectivos. Ahora bien, el EB se relaciona con Afr y los valores medios de éste son diferentes en extratensivos, ambiguales e introversivos, siendo mayores en los primeros y decreciendo correlativamente; así, si la puntuación de EB señala un estado de ambigüedad, el dato de Afr complementa dicha información mostrándonos una inclinación hacia el estado intraversivo. La presencia de una proporción afectiva baja manifiesta que estas personas prefieren no verse implicadas en los contextos emocionalmente cargados, lo que incrementa los problemas de descontrol, y no evitarán las situaciones que los exacerben, lo que evidencia sus dificultades en la relación interpersonal (Sendín, 2007). 
La proporción forma color (FC: $\mathrm{CF}+\mathrm{C}$ ) señala una puntuación mayor del lado izquierdo de la proporción frente al lado derecho. Este resultado confirma que los sujetos se guían más por sus emociones y por la satisfacción inmediata de estos.

De ahí que, la suma de C y CF (1.30) es próxima al de FC (1.47); por lo que el valor de FC no demuestra ser significativamente mayor; en tal sentido, ambas puntuaciones muestran la presencia de aspectos emocionales sobre los ideacionales; es decir, la conducta se deja llevar por la emocionalidad.

Los datos de la proporción afectiva discrepan parcialmente de los obtenidos en un estudio chileno con una población similar (Jiménez, 2009). En dicho estudio se obtuvo valoraciones bajas tanto en FC y CF; mientras que el presente estudio solo CF se muestra baja.

La presencia de color puro (C Pura) se observa ligeramente elevada, lo cual pone en evidencia la dificultad para controlar descargas afectivas intensas, bien porque son incapaces de amortiguar cognitivamente la descarga a causa de la gran intensidad de la experiencia emocional, o bien porque toman la decisión de dejar libre ese impulso de descarga en lugar de tomarse el trabajo de modularlo (Sendín, 2007).

La puntuación de las respuestas de espacio blanco (S) es elevado, esto señala que los evaluados acentúan los componentes más individuales. Ahora bien, esta puntuación no se encuentra asociada a valoraciones AG elevadas, por lo que es posible que los evaluados tiendan a reaccionar impulsivamente al entrar en acción, pero esta característica tiende a desaparecer externamente frente al entorno familiar, formando parte de su repertorio conductual.

\section{Indicadores del clúster de autopercepción}

La siguiente tabla muestra los indicadores que conforman el clúster de autopercepción, impresiones personales sobre uno mismo, que forman una especie de red de conceptos relacionados con las propias características. Resultan fácilmente accesibles al sujeto a través del pensamiento consciente, pero otras lo son parcialmente e incluso algunas pueden resultar totalmente inaccesibles a este tipo de conocimiento debido a que representan aspectos rechazables o conflictivos que tienden a ser suprimidos o negados (Sendín, 2007).

El índice de egocentrismo ( $3 r+(2) / R)$ se muestra moderadamente elevado, señalando una tendencia a centrase en sí mismos más de lo habitual; es decir, tienen una inusual preocupación por sí mismos y con frecuencia conllevan una marcada despreocupación por el mundo exteriory sus demandas reales. El autocentramiento no es sinónimo de una alta autoestima, si observamos los valores de Sum $V>0$ ( $\mathrm{Me}=3.67$ ) y $\mathrm{MOR}=1.17$, nos indican que la autovaloración que se asignan es bastante deficiente a pesar de su excesivo autocentramiento. Las personas autocentradas otorgan una exagerada prioridad a su propio punto de vista, tienen dificultades para ver las cosas desde otras perspectivas, les resulta difícil colocarse en la postura de los demás, evidenciándose asísu falta de empatía. Todo ello influye en su forma de procesar la información externa, en su manera de elaborar conceptos y en su capacidad para poder situarse en ópticas distintas a las suyas (Sendín, 2007). 
Tabla 3. Indicadores del clúster de autopercepción.

\begin{tabular}{lcc} 
& $M$ & $D E$ \\
\hline $3 r+(2) / R$ & 0.39 & 0.15 \\
Fr+Rf & 0.1 & 0.3 \\
SumV & 3.67 & 2.2 \\
FD & 0.13 & 0.34 \\
An+Xy & 1.64 & 2.25 \\
MOR & 1.17 & 1.24 \\
H: $(H)+H d+(H d)$ & $1.23: 1.70$ & $1.12: 1.55$ \\
Dd & 5.7 & 2.82 \\
\hline
\end{tabular}

Fuente: De la muestra de colaboradores del estudio.

Respecto al dato obtenido de las respuestas reflejas $(\mathrm{Fr}+\mathrm{rF})$, se encuentra una media $0.10(\mathrm{Me}=0.10)$, lo que revela un elemento dominante en el concepto que el grupo tiene de sí mismo. Esto conlleva una acusada tendencia a sobre estimar externamente la valía personal. Asimismo, se observa que $\mathrm{Fr}+\mathrm{rF}>0+\mathrm{Sum}$ $V>0$, estos datos evidencian que estas personas suelen tener problemas para establecer relaciones interpersonales profundas y significativas porque su propensión a negar así como a actuar en lugar de reflexionar, dificulta seriamente el compromiso afectivo. Por lo que, las estrategias defensivas funcionan con menos eficacia tornándose desadaptativas (Sendín, 2007).

La sumatoria de V+FD $>2$, indica que el grupo evaluado exagera las tareas de autoobservación, con consecuencias emocionales irritantes y con un excesivo aumento de su distancia con el entorno.

Pese a que la puntuación MOR es ligeramente superior a la hallada por Jiménez (2009), esta es aun así baja, lo cual supone que la autoimagen de estos sujetos está articulada con rasgos negativos, deteriorados o disfóricos. No obstante, la intensión de inclinarse por asumir una valoración positiva podría presentarse como un mecanismo defensivo frente a estados emocionales irritantes.

Dentro de las subcategorías de respuestas humanas, las respuestas de H pura son las más elevadas ( $\mathrm{Me}=1.23$ ), lo que implica una percepción centrada en la realidad que confirma que la muestra no presenta sintomatología psicopatológica. Próxima a $\mathrm{H}$ pura se encuentra $\mathrm{Hd}(\mathrm{Me}=1.10$ ) la cual señala un modo más cauteloso y suspicaz de acercamiento al otro. No obstante, si revisamos las fórmulas que combinan los diversos contenidos humanos, la proporción $\mathrm{H}:(\mathrm{H})+\mathrm{Hd}+(\mathrm{Hd})$ indica el predominio del lado derecho. Este dato nos indica la presencia de sujetos inmaduros, con actitudes suspicaces sobre los demás y con percepciones sesgadas en las relaciones interpersonales. Asimismo, el predominio de $\mathrm{Hd}$ en el lado derecho indica que el grupo tiene una percepción limitada y parcial del elemento humano (propio y ajeno) y tiende a mantener un estilo retraído y desconfiado en el contacto humano (Sendín, 2007). 
Por otro lado, revisando las puntuaciones GHR y PHR se observa que el segundo es superior a la primera; cuando se observa esto, las conceptualizaciones del grupo acerca de las relaciones interpersonales y de la autopercepción resultan menos efectivas para producir respuestas adaptativas; aumentando la posibilidad que se produzcan fallos o conflictos significativos en una o en ambas esferas de su funcionamiento.

Por último, el índice de estilo obsesivo (OBS) se presenta positivo, en función de la puntuación $\mathrm{Dd}>3(\mathrm{M}=5.70)$ que indica que el grupo en estudio parece necesitar intensamente hacerse valorar en lo personal, sin permitirse cometer algún error porque focaliza excesivamente suatención en los elementos negativos y tiende a magnificar la importancia de cualquier equivocación propia (Sendín, 2007).

Indicadores del clúster de relaciones interpersonales:

La siguiente tabla muestra los indicadores que conforman el clúster de relaciones interpersonales, uno de los elementos esenciales y constitutivos de la conducta humana (Sendín, 2007).

Respecto a la Proporción a: $p$, se observa que la puntuación a es significativamente mayor a $p$, lo cual indica que se trata de un grupo cognitivamente rígido; es decir, muestran poco interés a buscar soluciones nuevas o a adoptar pautas de conducta alternativas (Sendín, 2007).

Las puntuaciones de $\mathrm{p}$ y Fd son significativamente bajas por lo que se descarta la presencia de conductas dependientes. Estos resultados discrepan con los hallados en el estudio de Jiménez (2009) donde la puntuación p es ligeramente superior a la valoración a. En aquella muestra los delincuentes sexuales presentaban un tipo de vínculo en el que priman los contactos pasivos, mientras que en este estudio priman los contactos activos.

Tabla 4. Indicadores del clúster de relacione interpersonales.

\begin{tabular}{lcc}
\hline & M & DE \\
\hline A & 5.73 & 2.38 \\
P & 1.87 & 1.28 \\
GHP & 1.6 & 1.08 \\
PHR & 1.93 & 1.48 \\
Fd & 0.1 & 0.3 \\
Sum T & 0.43 & 0.88 \\
Contenido Humano & 3.27 & 2.39 \\
H Pura & 1.23 & 1.12 \\
PER & 3.93 & 5.01 \\
AG & 0.57 & 0.8 \\
COP & 0.33 & 0.83
\end{tabular}




\begin{tabular}{lll} 
Índice de aislamiento & 0.18 & 0.17 \\
$\mathrm{M}$ & 1.87 & 1.26 \\
$\mathrm{FM}$ & 4.77 & 2.19 \\
\hline
\end{tabular}

Fuente: De la muestra de colaboradores del estudio.

Respecto a GHP y PHR, Sendín (2007) refiere que lo habitual es encontrar un cómputo final de GHR > PHR; sin embargo, en esta investigación se observa que dicha relación no existe. PHR es ligeramente mayor a GHR lo cual señala que el grupo tiende a actuar con menos eficacia y adaptabilidad en el terreno interpersonal y suele ser percibida por los demás de manera poco favorable. Este predominio parece mostrar que los constructos de los sujetos sobre sí mismos y de los que los rodean son escasamente realistas y contienen excesivos sesgos personales. Esto lo convierte en un importante indicador de alteraciones en la autoimagen, en las representaciones mentales sobre los demás y en las conceptualizaciones acerca de los demás. Igualmente, en el estudio de Jiménez (2009) se aprecia que la muestra de delincuentes sexuales tiende a presentar una percepción distorsionada de la realidad, unido a un distanciamiento de lo convencional.

En la revisión de las puntuaciones PER, COP y AG, es evidente que la puntuación PER predomina sobre los otros dos datos, esta valoración muestra en el grupo una necesidad mayor de lo habitual de justificar defensivamente su autoimagen. En adultos señala una persona con mayor inseguridad de lo habitual y con tendencia a recurrir a un autoritarismo infantil, exigiendo que se le dé la razón cuando se siente puesta a prueba. Suelen ser vistas por lo demás como dogmáticos o rígidos y tienen dificultades interpersonales, sobre todo si su entorno no se somete a sus exigencias (Sendín, 2007). Este resultado también fue hallado en el estudio chileno ya mencionado anteriormente, en el que señalan que los delincuentes sexuales presentan un estilo autoritario de relación interpersonal. Por otro lado, los valores de COP y AG son bajos, lo cual indica que el grupo apenas realiza atribuciones en sus perceptos de interacción, no prevén aspectos positivos ni negativos en sus relaciones interpersonales. No están interesados a verse implicados en situaciones interpersonales, se presentan distantes o despegados. Igualmente, se aprecia dificultad para relacionarse de manera práctica tanto con elementos del entorno como con otras personas (Jiménez, 2009).

Ahora bien, si consideramos el déficit en la capacidad de control (mencionada en el apartado anterior), el bajo monto de recursos que no permiten satisfacer sus necesidades internas unido a la incapacidad de posponer dicha satisfacción de sus necesidades y controlar sus impulsos, los lleva a buscar alternativas inmediatas de gratificación.

Por otro lado, se observa que el valor del movimiento animal (FM) es mayor al de movimiento humano (M), lo cual muestra la disposición a regirse por sus necesidades de satisfacción inmediata más que por objetivos a largo plazo. Dicho predominio de FM sobre M se observa también el estudio de Jiménez (2009). 
En ambas investigaciones resalta la falta de empatía ( $\mathrm{M}$ bajo), esto puede entenderse en base a lo señalado por Friedlander en 1967, quien postuló que la falta de modelos y figuras significativas adecuadas -gratificadoras, cálidas, capaces de establecer límites claros-influye negativamente en la vida adulta del individuo. De esto se puede deducir que los delincuentes sexuales no aprendieron a vincularse ni a valorar la vida; así, el dañar una vida humana aparece como otra alternativa viable en la búsqueda de la satisfacción de sus necesidades y como una forma de validarse ante los demás.

\section{CONCLUSIONES Y RECOMENDACIONES}

1. Del estudio se concluye que la población infantil entre los 6 a 17 años es la más vulnerable a ser agredida sexualmente; siendo el agresor, en la mayoría de casos, el padre, padrastro o algún familiar cercano al entorno familiar. Asimismo, la población infantil femenina es la más vulnerable a ser agredida sexualmente; no obstante, los casos de agresión sexual al varón se han incrementado notablemente en los últimos años. La población afectada por violencia sexual ha aumentado en los últimos 10 años. En 2002 se registraron 1,023 mientras que a finales de 2012 se registraron 4,125 casos de violencia sexual contra niños, niñas y adolescentes entre 0 y 17 años.

2. Respecto a las características de personalidad, se encontró que el grupo de agresores sexuales intrafamiliares se caracteriza por: 1) Presentar escasos recursos para controlar descargas afectivas intensas. 2) Responder de manera impulsiva frente a descargas afectivas intensas. 3) No tener un estilo definido de respuesta básica y por ello, aleatoriamente, procesan afectos o tienden a usar la ideación, siendo mucho más impredictibles en su conducta. 4) Tener una autopercepción caracterizada por una autovaloración impregnada de matices de autocrítica negativa, tal desvalorización genera una estrategia defensiva de tipo narcisista para negar el dolor y la limitación.

5) Tener rasgos afectivos con estados de constricción emocional; es decir, presentan un freno en la expresión de sus emociones. 6) En lugar de registrar un alivio por la externalización o descarga de algún afecto, lo interiorizan y no permiten su salida lo que hace que su incomodidad interior se incremente. 7) Prefieren no verse implicados en contextos emocionalmente cargados, neutralizando los problemas de descontrol, si los hubiera, para evitar situaciones que los exacerben; esto puede sugerir dificultades en la relación interpersonal. 9) sus relaciones interpersonales se caracterizan por mostrar una tendencia a centrase en sí mismos más de lo habitual, por lo que tienen dificultades para ver las cosas desde otras perspectivas; además, presentan dificultades para establecer relaciones interpersonales profundas y significativas.

3. Finalmente, se recomienda seguir investigando sobre este tema en una muestra más amplia de agresores sexuales intrafamiliares, para poder validar lo encontrado en la presente investigación y poder profundizar en el estudio de las características de personalidad de esta población; además de contribuir a la construcción de un perfil del agresor sexual intrafamiliar. Asimismo, se insta a continuar desarrollando trabajos de investigación en el campo del abuso sexual intrafamiliar, puesto que se trata de un campo poco atendido y descuidado en la investigación psicológica de nuestro país. 
4. Por último, se sugiere abordar esta problemática con un equipo multidisciplinario a fin de abordar alcanzar una mirada más completa e integral que permita conocer los aspectos psicológicos, sociales, antropológicos, entre otros, de esta realidad.

\section{REFERENCIAS BIBLIOGRÁFICAS}

American Psychological Association (2010). Manual de publicaciones ( $3^{\circ}$ ed.). Washington, DC: El manual moderno.

Exner, J. (2005). Principios de interpretación del Rorschach. Manual para el sistema comprensivo. Madrid: Psimática.

Friedlander, K. (1967). Psicoanálisis de la delincuencia juvenil. Buenos Aires: Paidós.

Garrido, V. (2005) Qué es la psicología criminológica. Biblioteca Nueva. Madrid.

Hernández, R., Fernández, C. y Baptista. P. (2010). Metodología de la Investigación. ( $5^{\circ}$ ed.). México D. F.: McGraw-Hill.

Jiménez, P. (2009). Caracterización psicológica de un grupo de delincuentes Sexuales chilenos a través del test de Rorschach. La Frontera, Argentina. PSYKHE, 18, 27-38.

Kerlinger, F. (2002). Investigación del comportamiento. México D.F.: McGraw-Hill.

Organización mundial de la salud (2002). Informe mundial sobre la violencia y la salud. Ginebra. Suiza.

Programa Nacional Contra la Violencia Familiar y Sexual (2012). Resumen Estadístico Personas Afectadas por Violencia Familiar y Sexual atendidas CEM 2012: Lima: MIMP.

Programa Nacional Contra la Violencia Familiar y Sexual. (2013). Resumen Estadístico Personas Afectadas por Violencia Familiar y Sexual atendidas CEM 2013. Lima: MIMP.

Sendín, M. (2007). Manual de Interpretación del Rorschach para el Sistema Comprehensivo. Tercera Edición Revisada. Madrid: Psimática.

Viviano, T. (2012). Abuso sexual: estadísticas para la reflexión y pautas para la prevención. Programa Nacional contra la Violencia Familiar y Sexual. MIMP. 